\title{
A closure looks to Iris Recognition system
}

\author{
Sukhwinder Singh, Ajay Jatav \\ Assistant professor, at PEC Chandigarh, ME student at PEC Chandigarh,
}

\begin{abstract}
Iris Recognition Has Become A Popular Research In Recent Years. Due To Its Reliability And Nearly Perfect Recognition Rates, It Is Used In High Security Areas. Some Of The Applications Of Iris Recognition System Are Border Control In Airports And Harbors, Access Control In Laboratories And Factories, Identification For Automatic Teller Machines (Atms) And Restricted Access To Police Evidence Rooms. This Paper Provides A Review Of Major Iris Recognition Researches. There Are Four Main Stages In Iris Recognition System Namely: Segmentation, Normalization, Feature Extraction And Matching. A Literature Review Of The Most Prominent Algorithms Implemented Is Presented In This Paper And Lastly We Made A Compression Of Some More Iris Recognition Algorithms.
\end{abstract}

Keyword: Iris Recognition, Segmentation, Normalization, Features Extraction, Matching.

\section{INTRODUCTION}

Biometric authentication has been receiving extensive attention over the past decade with increasing demands in automated personal identification. The most popular biometric features are based on individuals' signature, retinal, faces, iris, fingerprints, hand and voices. Among all these biometric techniques, iris recognition is one of the most promising approaches due to its high reliability for personal identification.

The iris is a thin circular diaphragm, which lies between the cornea and the lens of the human eye. A front-on view of the iris is shown in Figure 1. The iris is perforated close to its centre by a circular aperture known as the pupil. The function of the iris is to control the amount of light entering through the pupil, and this is done by the sphincter and the dilator muscles, which adjust the size of the pupil. The average diameter of the iris is $12 \mathrm{~mm}$, and the pupil size can vary from $10 \%$ to $80 \%$ of the iris diameter.

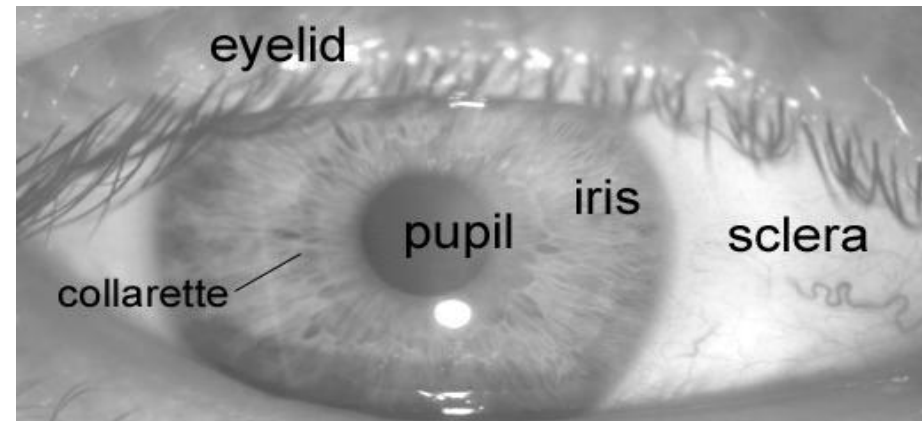

Figure 1. front view of human eye

The iris has unique features and complex enough to be used as biometric signature. This means that the probability of finding two people with identical iris pattern is almost zero. Image processing techniques can be employed to extract the unique iris pattern from a digitized image of the eye, and encode it into a biometric template, which can be stored in a database. This biometric template contains an objective mathematical representation of the unique information stored in the iris, and allows comparisons to be made between templates. When a subject wishes to be identified by an Iris recognition system, their eye is first photographed, and then a template created for their iris region. This template is then compared with the other templates stored in a database until either a matching template is found and the subject is identified, or no match is found and the subject remains unidentified.

John Daugman, was first implemented a working automated iris recognition system [1]. The Daugman system is patented [2] and the rights are now owned by the company Iridian Technologies. Even though the Daugman system is the most successful and most well known, many other systems have been developed. The most notable include the systems of Wildes et al. [3][4], Boles and Boashash [5], Lim et al. [6], and Noh et al. [7]. The algorithms by Lim et al. are used in the iris recognition system developed by the Ever media and Senex companies. Also, the Noh et al. algorithm is used in the 'IRIS2000' system, sold by IriTech. 


\section{DAUGMAN,S WORK}

In daugman's work [1] the visible texture of a person's in real-time video image is encoded into compact sequence of multi-scale quadrature 2-D Gabor Wavelet coefficients, whose most-significant bits comprise a 256-byte "iris code".

The final outcome of this work was a mathematical proof that there were sufficient degrees-offreedom, or form of variation in the iris among individuals, to impart to it the same singularity as a conventional fingerprint. Also uncertain was whether efficient algorithms could be developed to extract a detailed iris description reliably from a live video image, generate a compact code for the iris (of minuscule length compared with image data size), and render a decision about individual identity with high statistical confidence. The final problem was whether the algorithms involved could be executed in real time on a general-purpose microprocessor. During his work all of these question were resolved and a working system was described.

Daugman's work comprises four main parts namely

1- Segmentation.

2- Normalization.

3- Feature extraction.

4- Matching.

\subsection{Segmentation}

Daugman makes use of an integro-differential operator for locating the circular iris and pupil regions, and also the arcs of the upper and lower eyelids. The integro-differential operator is defined as

$$
\max _{\left(r, x_{0}, y_{0}\right)}\left|G_{\sigma}(r) * \frac{\partial}{\partial r} \oint_{r, x_{0} y_{0}} \frac{I(x, y)}{2 \pi r} d s\right|
$$

whereI $(\mathrm{x}, \mathrm{y})$ is the eye image, $\mathrm{r}$ is the radius to search for, $G_{\sigma}(r)$ is a Gaussian smoothing function, and $\mathrm{s}$ is the contour of the circle given by $\mathrm{r}, x_{0}, y_{0}$. The operator searches for the circular path where there is maximum change in pixel values, by varying the radius and centre $\mathrm{x}$ and $\mathrm{y}$ position of the circular contour. The operator is applied iteratively with the amount of smoothing progressively reduced in order to attain precise localization. Eyelids are localized in a similar manner, with the path of contour integration changed from circular to an arc.

\subsection{Normalization}

For normalization Daugman's[1] invented rubber sheet model in which he remaps each point within the iris region to a pair of polar coordinates $(r, \theta)$ where $r$ is on the interval $[0,1]$ and $\theta$ is angle $[0,2 \pi]$.
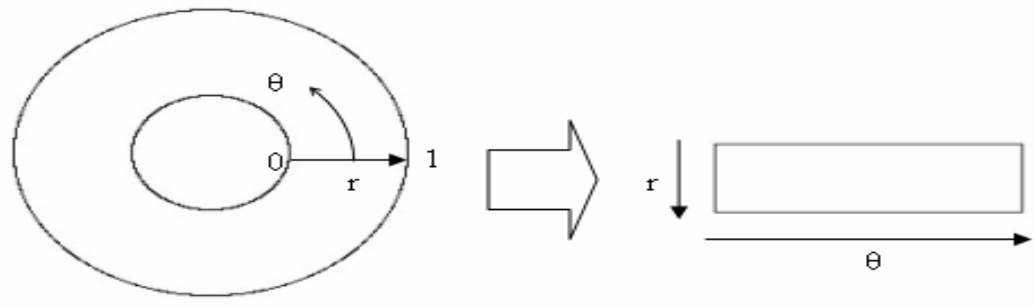

Figure 2. Daugman's rubber sheet model

The remapping of the iris region from $(x, y)$ Cartesian coordinates to the normalised non-concentric polar representation is modeled as

With

$$
I(x(r, \theta), y(r, \theta)) \rightarrow I(r, \theta)
$$

$$
\begin{aligned}
& x(r, \theta)=(1-r) x_{p}(\theta)+r x_{i}(\theta) \\
& y(r, \theta)=(1-r) y_{p}(\theta)+r y_{i}(\theta)
\end{aligned}
$$

where $I(x, y)$ is the iris region image, $(x, y)$ are the original Cartesian coordinates, $(r, \theta)$ are the corresponding normalized polar coordinates, $x_{p}, y_{p}$ and $x_{i}, y_{i}$ are the coordinates of the pupil and iris boundaries along the $\theta$ direction. The rubber sheet model takes into account pupil dilation and size inconsistencies in order to produce a normalized representation with constant dimensions. In this way the iris region is modeled as a flexible rubber sheet anchored at the iris boundary with the pupil centre as the reference point. 


\section{FEATURE ENCODING}

Daugman makes uses of a 2D version of Gabor filters [1] in order to encode iris pattern data. A 2D Gabor filter over the an image domain $(x, y)$ is represented as

$$
\mathrm{G}(\mathrm{x}, \mathrm{y})=\mathrm{e}^{-\pi\left[\left(\mathrm{x}-\mathrm{x}_{0}\right)^{2} / \mathrm{x}^{2}+\left(\mathrm{y}-\mathrm{y}_{0}\right)^{2} / \beta^{2}\right]} \mathrm{e}^{-2 \pi \mathrm{i}\left[\mathrm{u}_{0}\left(\mathrm{x}-\mathrm{x}_{0}\right)+\mathrm{v}_{0}\left(\mathrm{y}-\mathrm{y}_{0}\right)\right]}
$$

Where $\left(x_{0}, y_{0}\right)$ specify position in the image, $(\alpha, \beta)$ specify the effective width and length, and $\left(u_{0}, v_{0}\right)$ specify modulation, which has spatial frequency $\omega_{0}=\sqrt{u_{0}^{2}+v_{0}^{2}}$ The odd symmetric and even symmetric 2D Gabor filters are shown in Figure 3

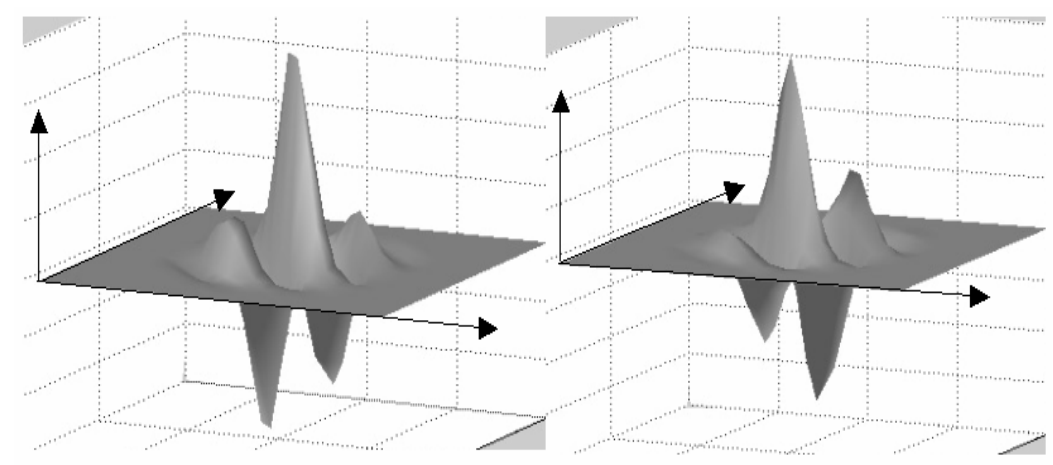

Figure 3.

A quadrature pair of 2D Gabor filters left) real component or even symmetric filter characterized by a cosine modulated by a Gaussian right) imaginary component or odd symmetric filter characterized by a sine modulated by a Gaussian

Daugman demodulates the output of the Gabor filters in order to compress the data. This is done by quantizing the phase information into four levels, for each possible quadrant in the complex plane. Taking only the phase will allow encoding of discriminating information in the iris, while discarding redundant information such as illumination, which is represented by the amplitude component.

These four levels are represented using two bits of data, so each pixel in the normalized iris pattern corresponds to two bits of data in the iris template. A total of 2,048 bits are calculated for the template, and an equal number of masking bits are generated in order to mask out corrupted regions within the iris. This creates a compact 256-byte template, which allows for efficient storageand comparison of irises. The Daugman system makes use of polar coordinates for normalization, therefore in polar form the filters are given as

$$
H(r, \theta)=e^{-\omega\left(\theta-\theta_{0}\right)} e^{-\left(r-r_{0}\right)^{2} / \alpha^{2}} e^{-i\left(\theta-\theta_{0}\right)^{2} / \beta^{2}}
$$

Where $(\alpha, \beta)$ are the same as describe earlier, and $\left(r_{0}, \theta_{0}\right)$ specify the centre frequency of the filter.

\subsection{Matching}

Duagman's use hamming distance a matching metric developed by him, and calculation of the Hamming distance is taken only with bits that are generated from the actual iris region.

The Hamming distance gives a measure of how many bits are the same between two bit patterns. Using the Hamming distance of two bit patterns, a decision can be made as to whether the two patterns were generated from different irises or from the same one.

In comparing the bit patterns $X$ and $Y$, the Hamming distance, $H D$, is defined as the sum of disagreeing bits (sum of the exclusive-OR between $X$ and $Y$ ) over $N$, the total number of bits in the bit pattern.

$$
H D=\frac{1}{N} \sum_{j=1}^{N} X_{j}(X O R) Y_{j}
$$

Since an individual iris region contains features with high degrees of freedom, each iris region will produce a bit-pattern which is independent to that produced by another iris, on the other hand, two iris codes produced from the same iris will be highly correlated.

If two bits patterns are completely independent, such as iris templates generated from different irises, the Hamming distance between the two patterns should equal 0.5. This occurs because independence implies the two bit patterns will be totally random, so there is 0.5 chance of setting any bit to 1 , and vice versa. Therefore, half of the bits will agree and half will disagree between the two patterns. If two patterns are derived from the 
same iris, the Hamming distance between them will be close to 0.0 , since they are highly correlated and the bits should agree between the two iris codes.

\section{BOLES' WORK}

The boles proposed a iris recognition system which is designed to handle noisy condition as well as possible variation in illumination and camera-to-face distance. His work is based on zero crossing of wavelet transform. In his work apart from the iris location a normalization algorithm brings the iris to have the same diameter and the same number of data points. From the grey levels of the sample images one-dimentional signals are obtained and referred to as the iris signature. Then a zero-crossing representation is calculated based on the wavelet transform. These representations are stored ax templets and are used for the matching algorithm. In this way the auther claims that the noise influence will be eliminated since zero crossing campared to data points could speed the computations. An interesting aspect of this system is the ability to wavelet transform to eliminate glares due to reflection of the light source on the surface of the iris.

Boles's work comprises mainly four parts namely

1. Segmentation

2. Normalization

3. Feature extraction and

4. Matching

\subsection{Segmentation}

The process of information extraction starts by locating the pupil of the eye, which can be done using any edge detection technique. Knowing that it has a circular shape, the edges defining it are connected to form a closed contour. The grey level values on the contour of virtual concentric circles, which are centered at the centroid of the pupil, are recorded and stored in circular buffersIn what follows, for simplicity, one such data set will be used to explain the process and will be referred to as the iris signature.

Using the edge-detected image, the maximum diameter of the iris in any image is calculated. In comparing two images, one will be considered to be a reference image. The ratio of the maximum diameter of the iris in this image to that of the other image is also calculated. This ratio is then used to make the virtual circles, which extract the iris features, have the same diameter. In other words, the dimensions of the irises in the images will be scaled to have the same constant diameter regardless of the original size in the images.

\subsection{Normalization}

In the Boles [4] system, iris images are first scaled to have constant diameter so that when comparing two images, one is considered as the reference image. This works differently to the other techniques, since normalization is not performed until attempting to match two iris regions, rather than performing normalization and saving the result for later comparisons. Once the two irises have the same dimensions, features are extracted from the iris region by storing the intensity values along virtual concentric circles, with origin at the centre of the pupil. A normalization resolution is selected, so that the number of data points extracted from each iris is the same. This is essentially the same as Daugman's rubber sheet model, however scaling is at match time, and is relative to the comparing iris region, rather than scaling to some constant dimensions. Also, it is not mentioned by Boles, how rotational invariance is obtained.

\subsection{Feature extraction}

Boles and Boashash [5] make use of 1D wavelets for encoding iris pattern data. The mother wavelet is defined as the second derivative of a smoothing function $\theta(x)$.

$$
\psi(x)=\frac{d^{2} \theta(x)}{d x^{2}}
$$

The zero crossings of dyadic scales of these filters are then used to encode features. The wavelet transform of a signal $f(x)$ at scale $s$ and position $x$ is given by

$$
\begin{gathered}
w_{s} f(x)=f *\left(s^{2} \frac{d^{2} \theta(x)}{d x^{2}}\right)(x) \\
=s^{2} \frac{d^{2}}{d x^{2}}\left(f * \theta_{s}\right)(x)
\end{gathered}
$$

$$
\theta_{s}=(1 / s) \theta(x / s)
$$

$w_{s} \mathrm{f}(\mathrm{x})$ is proportional to the second derivative of $\mathrm{f}(\mathrm{x})$ smoothed by $\theta_{s}(\mathrm{x})$, and the zero crossings of the transform correspond to points of inflection in $f * \theta_{S}(\mathrm{x})$. The motivation for this technique is that zero-crossings correspond to significant features with the iris region. 


\subsection{Matching}

Boles and Boashash proposed a modal based algorithm in which the original signature of different irises to be recognized are represented by their zero-crossing representations. These representations are then stored in the database of the system and are referred to as models. The main task is to match an iris in an image, which is referred to as an unknown, with one of the models whose representations are stored in the database.

In order to classify unknown signatures, they consider two alternative dissimilarity functions that compare the unknown signature $g$ and candidate model $f$ at a particular resolution level $j$ as

$$
\begin{gathered}
d_{j}^{(1)}(f, g)=\min _{m} \sum_{n=1}^{N}\left|Z_{j} f(n)-\Gamma Z_{\mathrm{j}} \mathrm{g}(\mathrm{n}+\mathrm{m})\right|^{2} \\
m \in[0, \quad N-1] \\
d_{j}^{(2)}(f, g)=\min _{m} \frac{\sum_{r=1}^{R_{j}}\left\{\left[\mu_{j}(r)\right]_{f}\left[\rho_{j}(r)\right]_{f}-\Gamma\left[\mu_{j}(r+m)\right]_{g}\left[\rho_{j}(r+m)\right]_{g}\right\}^{2}}{\Gamma \sum_{r=1}^{R_{j}}\left|\left[\mu_{j}(r)\right]_{f}\left[\rho_{j}(r)\right]_{f}\right|\left|\left[\mu_{j}(r)\right]_{g}\left[\rho_{j}(r)\right]_{g}\right|} \\
m \in\left[0, \quad R_{j}-1\right]
\end{gathered}
$$

Where $\Gamma$ is the scale factor and equals the ratio between the radius of virtual circle of the candidate model and that of unknown signature. Note that the first dissimilarity function is computed using every point of the representation, whereas the second uses only the zero-crossing points. The overall dissimilarity value over the resolution interval $[\mathrm{K} ; \mathrm{L}]$ will be the average of the dissimilarity functions calculated at each resolution level in this interval, i.e.,

$$
D^{(p)}=\sum_{K}^{L} \frac{d_{j}^{(p)}(f, g)}{Q}
$$

wherep $=1,2$ refers to one of the dissimilarity functions defined above. The first dissimilarity function $d_{j}^{1}$ makes a global measurement of the difference of energy between two zero-crossing representations over the entire spatial domain at a particular level $\mathrm{j}$.In order to grasp the most essential information selectively and effectively and to simplify the computation, they introduce the other dissimilarity function $d_{j}^{(2)}$, which compares two representations basedon the dimensions of the rectangular pulses of the zero-crossing representations. With this function, the amount of computation is significantly reduced since the number of zero crossings is much less than the total number of data points. However, the main problem of using this function is that it requires the compared representations to have the same number of zero crossings at each resolution level. To overcome this problem, one can do either of the following.

a) Check the number of zero crossings of the representations of the unknown signature and candidate model. If the above condition is satisfied for at least two adjacent resolution levels, then use $d_{j}^{2}$. The overall dissimilarity value $D^{(2)}$ is calculated in the interval where the number of zero-crossings is the same.

b) Alternatively, use a false zero-crossing elimination algorithm to remove unimportant (or false) zero crossings in order to make the number of zero crossings of the unknown and model representations be the same before calculating the dissimilarity function.

\section{COMPARISON}

The first system was awarded US patents. During the evolvement process of Daugman's(1,2) system perfect result were reported testing 592 irises. However the few shortcoming of daugman's system are During segmentation the duagman's Integro-differential algorithm fail where there is noise in the eye image, such as from reflections, since it works only on a local scale.

Even though the homogenous rubber sheet model accounts for pupil dilation, imaging distance and nonconcentric pupil displacement, it does not compensate for rotational inconsistencies. In the Daugman system, rotation is accounted for during matching by shifting the iris templates in the $\theta$ direction until two iris templates are aligned.

Boles(5) tried to implement a system extending the current capabilities of the existing methods. He basically tries to create a system that would not need irises being in the same location within the image of glare free under fixed illuminations. This problem was already solved in daugman's project by the sequential processing of various frames until the edge detector for the iris location reported that an iris was present.

Nevertheless, the wavelet transform provide the ability of pattern matching under local distortion hoping to compensate for glare resulting from reflection of light on the surface of the iris and translation and size variations. He also tries to test the tolerance of his system in different noise levels. Even though the results were good the system was tested with a very small number of images that limits the further conclusions. Something that differentiates his work is the analysis of the information in the various resolution levels. In his 
attempt to degrease the computational needs he discovered that intermediate levels contained most of the energy.

\section{Table 1. Comparision table of some proposed iris recognition algorithim}

\begin{tabular}{|c|c|c|c|c|}
\hline $\begin{array}{l}\text { Research } \\
\text { papers }\end{array}$ & Iris segmentation & $\begin{array}{l}\text { Image } \\
\text { enhancement }\end{array}$ & Feature extraction and matching & Additional comments \\
\hline $\begin{array}{l}\text { Daugman } \\
(1,2)\end{array}$ & $\begin{array}{l}\text { Integro-differential } \\
\text { operator }\end{array}$ & - & $\begin{array}{l}\text { 2-D gabor transform thamming } \\
\text { distance }\end{array}$ & $\begin{array}{l}\text { First iris recognition } \\
\text { algorithm }\end{array}$ \\
\hline Wildes (3) & $\begin{array}{l}\text { Image intensity gradiant } \\
\text { and hough transform }\end{array}$ & - & $\begin{array}{l}\text { Lapplacian of } \\
\text { gaussionfilters -normalizedcorrellation }\end{array}$ & \\
\hline $\begin{array}{l}\text { Boles and } \\
\text { Boashash }(5)\end{array}$ & Edge detection & - & $\begin{array}{l}\text { Wavelet transform zero crossing - } \\
\text { dissimilarity function }\end{array}$ & $\begin{array}{l}\text { Does not perform for } \\
\text { non idial iris images }\end{array}$ \\
\hline Ma et al $(8)$ & $\begin{array}{l}\text { Grey level information } \\
\text { and canny edge detector }\end{array}$ & $\begin{array}{l}\text { Back ground } \\
\text { subtraction }\end{array}$ & $\begin{array}{l}\text { Multichannel spatial filter-fisher- } \\
\text { linear discriminate classification }\end{array}$ & $\begin{array}{l}\text { Does not work with } \\
\text { occluded images }\end{array}$ \\
\hline $\begin{array}{l}\text { Avila and } \\
\text { Reillo (9) }\end{array}$ & Intensity based ditection & - & $\begin{array}{l}\text { Gabor filter and multiscalezero- } \\
\text { crossing-Euclidean and hamming } \\
\text { distanse }\end{array}$ & $\begin{array}{l}\text { Does not unwrap the } \\
\text { iris images }\end{array}$ \\
\hline $\begin{array}{l}\text { Vasta et } \\
\text { al.(10) }\end{array}$ & Intensity based ditection & - & $\begin{array}{l}\text { 1.D log Gabor and eular number } \\
\text { thamming distance and L1 distance }\end{array}$ & $\begin{array}{l}\text { Rule based decision } \\
\text { strategy is used to } \\
\text { improve accuracy }\end{array}$ \\
\hline $\begin{array}{l}\text { Monioet } \\
\text { al.(11) }\end{array}$ & $\begin{array}{l}\text { Heuristic gray level edge } \\
\text { feature }\end{array}$ & $\begin{array}{l}\text { Back ground } \\
\text { subtraction }\end{array}$ & 1.D DCT+ hamming distance & $\begin{array}{l}\text { Fast feature extraction } \\
\text { and matching }\end{array}$ \\
\hline $\begin{array}{l}\text { Poursaberi } \\
\text { and } \\
\text { Araabi.(12) }\end{array}$ & $\begin{array}{l}\text { Morphological operator } \\
\text { and thesholds }\end{array}$ & $\begin{array}{l}\text { Wiener 2D } \\
\text { filter }\end{array}$ & $\begin{array}{l}\text { Daubechies } 2 \text { Wavelet - hamming } \\
\text { distance and harmonic mean }\end{array}$ & \\
\hline $\begin{array}{l}\text { Daugman. } \\
\text { (13) }\end{array}$ & $\begin{array}{l}\text { Active countours and } \\
\text { generalized Coordinates }\end{array}$ & $\cdot$ & Iris codes & $\begin{array}{l}\text { Gaze deviation and } \\
\text { correction, second rank } \\
\text { in ICE } 2006 \text { and low } \\
\text { time complexity }\end{array}$ \\
\hline
\end{tabular}

\section{CONCLUSION}

Iris identification was considered one of the most robust ways to identify humans. It provides enough Degrees-of-Freedom for accurate and safe recognition. Iris is considered the most unique, data rich physical structure on the human body. It works even when people were sunglasses or contact lenses.

The properties of the iris that enhance its suitability for use in automatic identification include its natural protection from the external environment, impossibility of surgically modifying without the risk of vision, physiological response to light that is unique, permanent features and ease of registering its image at some distance. Iris Recognition Systems involve acquiring a picture of a person's iris, digitally encode it, and compare it with one already on file. Iris identification provides convenience to the human users and is widely accepted.

Dr. John Daugman developed the theoretical research and algorithms. Theoretically the problem involves edge detection operators (locate the iris), representation and correspondence function (the same parts of the different irises need to be compared, and a matching algorithm. The information contained in the iris structure lies throughout its frequency spectrum. Small and large structures, small and large edges are all distinguishable features (Freckles, Contractile Furrow). In order to exploit this, 2-D decomposition (Gabor Phasor Coefficients, Wavelets) is applied. The frequency components of the various bands are used to extract feature vectors suitable for robust recognition. The main problems that had to be solved apart from common computer vision drawbacks (scaling, illumination, and rotation) were the reflections of the eye to the light source.

The main disadvantage of Iris Recognition was the designing and manufacturing cost of such a system. 


\section{REFERENCES}

[1]. J. Daugman. How iris recognition works. Proceedings of 2002 International Conference on Image Processing, Vol.1, 2002.

[2]. J. Daugman. Biometric personal identification system based on iris analysis. United States Patent, Patent Number: 5,291,560, 1994.

[3]. R. Wildes, J. Asmuth, G. Green, S. Hsu, R. Kolczynski, J. Matey, S. McBride. A system for automated iris recognition. Proceedings IEEE Workshop on Applications of Computer Vision, Sarasota, FL, pp. 121-128, 1994.

[4]. R. Wildes. Iris recognition: an emerging biometric technology. Proceedings of the IEEE, Vol. 85, No. 9, 1997.

[5]. W. Boles, B. Boashash. A human identification technique using images of the iris and wavelet transform. IEEE Transactions on Signal Processing, Vol. 46, No. 4, 1998.

[6]. S. Lim, K. Lee, O. Byeon, T. Kim. Efficient iris recognition through improvement of feature vector and classifier. ETRI Journal, Vol. 23, No. 2, Korea, 2001.

[7]. S. Noh, K. Pae, C. Lee, J. Kim. Multiresolution independent component analysis for iris identification. The 2002 International Technical Conference on Circuits/Systems, Computers and Communications, Phuket, Thailand, 2002.

[8]. L. Ma, T. Tan, Y. Wang, and D. Zhang, "Personal identification based on iris texture analysis," IEEE Trans. Pattern Anal. Mach. Intell., vol. 25, no. 12, pp. 1519-1533, Dec. 2003.

[9]. C. Sanchez-Avila and R. Sanchez-Reillo, "Two different approaches for iris recognition using Gabor filters and multiscale zero-crossing represen-tation," Pattern Recognit., vol. 38, no. 2, pp. 231-240, Feb. 2005.

[10]. M. Vatsa, R. Singh, and A. Noore, "Reducing the false rejection rate of iris recognition using textural and topological features," Int. J. SignalProcess., vol. 2, no. 1, pp. 66-72, 2005.

[11]. D. M. Monro, S. Rakshit, and D. Zhang, "DCT-based iris recognition," IEEE Trans. Pattern Anal. Mach. Intell., vol. 29, no. 4, pp. 586-596, Apr. 2007.

[12]. A. Poursabery and B. N. Araabi, "Iris recognition for partially occluded images: Methodology and sensitivity analysis,” EURASIP J.Adv. Signal Process., vol. 2007, no. 1, p. 20, Jan. 2007. ArticleID 36751.

[13]. J. Daugman, "New methods in iris recognition," IEEE Trans. Syst., Man,Cybern. B, Cybern., vol. 37, no. 5, pp. 1168-1176, Oct. 2007. 